Article

\title{
Scoring Amino Acid Mutations to Predict Avian-to-Human Transmission of Avian Influenza Viruses
}

\author{
Xiaoli Qiang ${ }^{1}$, Zheng Kou ${ }^{1, *}$, Gang Fang ${ }^{1}$ and Yanfeng Wang ${ }^{2}$ \\ 1 Institute of Computing Science and Technology, Guangzhou University, Guangzhou 510006, China; \\ qiangxl@mail.scuec.edu.cn (X.Q.); yuxiangqd@163.com (G.F.) \\ 2 Henan Key Lab of Information-Based Electrical Appliances, College of Electrical and Electronic Engineering, \\ Zhengzhou University of Light Industry, Zhengzhou 450002, China; wangyanfeng@zzuli.edu.cn \\ * Correspondence: kouzhengcn@foxmail.com; Tel.: +86-20-3936-6191
}

Received: 17 May 2018; Accepted: 19 June 2018; Published: 29 June 2018

\begin{abstract}
Avian influenza virus (AIV) can directly cross species barriers and infect humans with high fatality. Using machine learning methods, the present paper scores the amino acid mutations and predicts interspecies transmission. Initially, 183 signature positions in 11 viral proteins were screened by the scores of five amino acid factors and their random forest rankings. The most important amino acid factor (Factor 3 ) and the minimal range of signature positions (50 amino acid residues) were explored by a supporting vector machine (the highest-performing classifier among four tested classifiers). Based on these results, the avian-to-human transmission of AIVs was analyzed and a prediction model was constructed for virology applications. The distributions of human-origin AIVs suggested that three molecular patterns of interspecies transmission emerge in nature. The novel findings of this paper provide important clues for future epidemic surveillance.
\end{abstract}

Keywords: avian influenza virus; interspecies transmission; amino acid mutation; machine learning

\section{Introduction}

Wild birds are regarded as the natural reservoir of avian influenza virus (AIV) [1]. Interspecies transmission might have been enabled long ago, when wild birds were domesticated by humans. A highly pathogenic subtype of AIV, avian influenza H5N1, originated in Asia in 1996 [2]. Human-origin H5N1 virus was first isolated from clinical samples in 1997, confirming that the H5N1 virus can directly cross species barriers and fatally infect the respiratory system [3,4]. Human infection by $\mathrm{H} 5 \mathrm{~N} 1$ has been continuously reported since 2003, attracting the attention of both researchers and wider society [5-8]. Moreover, viral subtypes other than H5N1 can infect humans by direct interspecies transmission. Two infectious cases of H9N2 virus have been reported; one in 1999, the other in 2003 [9,10]. H7N7 virus infected farmers in the Netherlands in 2003 [11], and H7N9 has continuously infected China's population since 2013 [12,13].

Interspecies transmission of AIV from its natural reservoir occurs in three steps: (1) the residence of AIVs in their wild animal hosts; (2) AIV contact with humans and direct infection with low probability; and (3) adaptation of AIVs to their new host and efficient human-to-human transmission thereafter. Thus far, AIV has not progressed beyond step 2, which represents initial adaption to the new host and low efficiency of transmission among the new host. The subtype viruses that can cross the species barrier and cause epidemics should be identified. Approximately twenty years has passed since human-originated AIV was first isolated from human samples in 1997. During this period, vast amounts of genomic data have accumulated in public databases. Therefore, after screening the 
important amino acid sites in the 11 viral proteins, the AIV risk can be predicted by machine learning methods and other mathematic models in the field of bioinformatics [14-18].

AIV transmission relies on amino acid mutations [19-21]. In a previous study, five amino acid factors (AA factors) summarized from 491 highly redundant amino acid attributes were associated with specific physiochemical amino acid properties, namely, polarity, secondary structure, molecular volume, codon diversity, and electrostatic charge [22]. In this paper, we use five AA factors to transform viral proteins and use the random forest (RF) method to select features from high-dimensional protein data and score them by their contributions to the data category. After ranking and screening the positions containing important mutation information, the classifier can predict the interspecies transmission phenotypes.

Two prediction models of AIVs have been published in the literature [23-25]. However, both of these models lack the protein data of hemagglutinin (HA) and neuraminidase (NA), and the biological meanings of the features were not clarified. To construct a more robust and meaningful model, we revise these models and screen the signature amino acid positions in HA, NA, and nine other viral proteins. To this end, we first identify 183 signature mutation positions by RF scoring, then predict AIV occurrence by four popular machine learning methods. Using the most effective classifier, we seek the important amino acid factors and the minimal range of signature positions. The study results will benefit epidemic surveillance and future studies on interspecies AIV transmission.

\section{Results}

\subsection{Dataset}

The cleaned dataset contained 869 high-quality AIV strains: 440 avian-origin AIVs (negative samples; H1-H14, H16 subtypes) and 429 human-origin AIVs (positive samples; H5N1, H5N6, H7N3, H7N7, H7N9, and H9N2 subtypes). The information related to these strains is summarized in Table S1.

\subsection{Signature Amino Acid Residues}

The importance score at each position in the 11 viral proteins was computed by RF. As shown in Figure 1a, the slope of the curve suddenly changes at an importance score of 9 . Therefore, 9 was selected as the cutoff score, providing 183 signature positions for further machine learning.
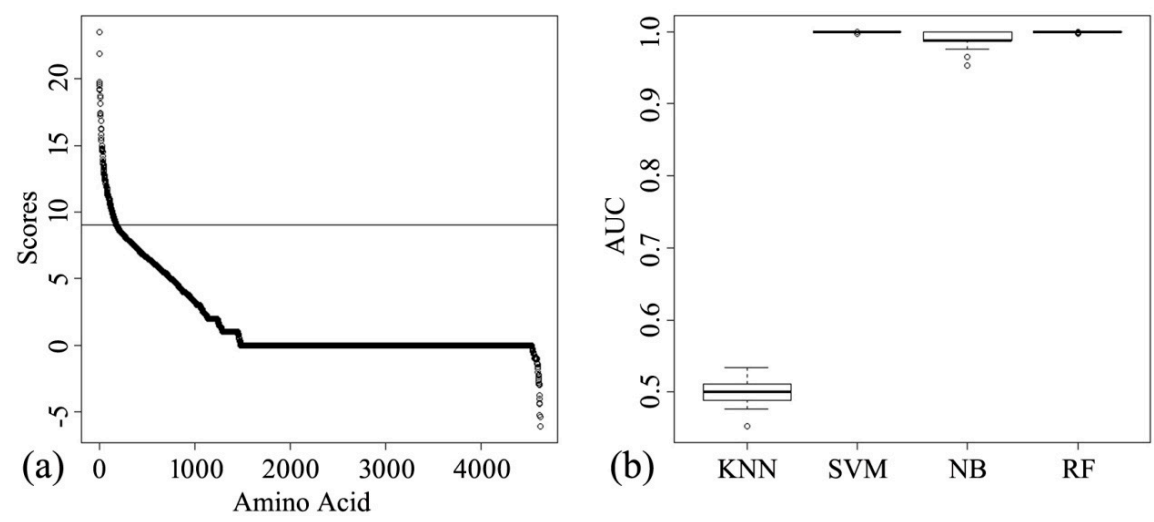

Figure 1. Importance score curve and the performances of $k$-nearest neighbor $(\mathrm{KNN})$, support vector machine (SVM), naïve Bayes (NB), and random forest (RF) classifiers. (a) The ranked scores were calculated from five AA factors using the random forest method. The $x$ and $y$ coordinates denote the total length of the 11 protein alignments and the importance scores, respectively. The cutoff value (9) is indicated by the thin horizontal line. (b) Performances of the four classifiers were evaluated from 100 repeats of 10 -fold cross-validation. The area under the curve (AUC) ranges from 0 to 1. 
As shown in Table 1, the HA protein contained the largest number of signature positions (65 amino acid residues), suggesting that HA is very important for interspecies transmission of AIVs. HA is mainly involved in receptor-binding and fusion activities. Positions HA102-HA290 (Table 1) locate in or close to the region of host receptor binding [26,27], and $\mathrm{H} 163$ is reportedly related to the specificity of receptor binding [28]. HA91, HA96, HA328, HA377, and HA397 locate at or near the fusion peptide [29], which triggers fusion activity in acidic environments and favors transmission to humans. The four HA327 positions located in the cleavage site are important virulence sites [30].

NA protein contains 44 signature positions (Table 1). The three NA52s located in the stalk deletion region are related to the virulence and pathogenesis of H5N1 influenza A virus [31]. NA19-NA37 located in the N-terminal are associated with structural stability and enzyme activity [32]. The PB2 627 position has been implicated in increased replication or virulence of AIVs in mammals [33]. PB1 14, located in the binding region of polymerase, is related to viral genome replication [34]. M2 97, which is affiliated with viral particle ensembles [35], was also screened. NEP 14, NP 373, and NP 377 are reportedly involved in intracellular transport of viral proteins [36,37].

Table 1. Scores for the 183 signature amino acids of avian influenza viruses (AIVs).

\begin{tabular}{|c|c|c|c|c|c|c|c|c|c|c|c|}
\hline Num & Pro $^{1}$ & $\operatorname{Pos}^{2}$ & Score & Num & Pro & Pos & Score & Num & Pro & Pos & Score \\
\hline 1 & PB2 & 389 & 11.95 & 62 & HA & 176 & 13.61 & 123 & NA & 65 & 10.98 \\
\hline 2 & PB2 & 478 & 9.81 & 63 & HA & 179 & 10.08 & 124 & NA & 66 & 9.93 \\
\hline 3 & PB2 & 598 & 17.36 & 64 & HA & 185 & 14.73 & 125 & NA & 72 & 10.96 \\
\hline 4 & PB2 & 627 & 9.83 & 65 & HA & 189 & 14.55 & 126 & NA & 79 & 11.38 \\
\hline 5 & PB2 & 648 & 15.55 & 66 & HA & 207 & 9.49 & 127 & NA & 85 & 9.57 \\
\hline 6 & PB2 & 676 & 9.94 & 67 & HA & 211 & 11.15 & 128 & NA & 88 & 10.13 \\
\hline 7 & PB1 & 14 & 19.16 & 68 & HA & 213 & 11.40 & 129 & NA & 100 & 11.34 \\
\hline 8 & PB1 & 48 & 18.13 & 69 & HA & 216 & 12.17 & 130 & NA & 187 & 10.48 \\
\hline 9 & PB1 & 113 & 18.58 & 70 & HA & 221 & 10.57 & 131 & NA & 205 & 9.62 \\
\hline 10 & PB1 & 149 & 11.09 & 71 & HA & 222 & 9.02 & 132 & NA & 233 & 10.13 \\
\hline 11 & PB1 & 257 & 13.74 & 72 & HA & 240 & 17.36 & 133 & NA & 249 & 9.05 \\
\hline 12 & PB1 & 383 & 12.14 & 73 & HA & 251 & 16.26 & 134 & NA & 257 & 17.24 \\
\hline 13 & PB1 & 384 & 9.34 & 74 & HA & 266 & 10.96 & 135 & NA & 265 & 9.29 \\
\hline 14 & PB1 & 387 & 11.50 & 75 & HA & 273 & 12.53 & 136 & NA & 285 & 10.46 \\
\hline 15 & PB1 & 525 & 9.95 & 76 & HA & 274 & 9.23 & 137 & NA & 287 & 10.65 \\
\hline 16 & PB1 & 573 & 13.38 & 77 & HA & 275 & 9.38 & 138 & NA & 288 & 10.28 \\
\hline 17 & PB1 & 628 & 9.59 & 78 & HA & 289 & 10.36 & 139 & NA & 333 & 10.07 \\
\hline 18 & PB1-F2 & 4 & 9.38 & 79 & HA & 290 & 11.74 & 140 & NA & 338 & 9.02 \\
\hline 19 & PB1-F2 & 26 & 9.24 & 80 & HA & 297 & 10.48 & 141 & NA & 347 & 9.82 \\
\hline 20 & PB1-F2 & 48 & 13.50 & 81 & HA & 315 & 11.98 & 142 & NA & 359 & 10.08 \\
\hline 21 & PB1-F2 & 50 & 11.81 & 82 & HA & 323 & 13.04 & 143 & NA & 368 & 11.05 \\
\hline 22 & PB1-F2 & 57 & 16.85 & 83 & HA & 327 & 12.84 & 144 & NA & 369 & 10.82 \\
\hline 23 & PB1-F2 & 77 & 11.29 & 84 & HA & 327 & 16.23 & 145 & NA & 399 & 11.71 \\
\hline 24 & PA & 37 & 18.74 & 85 & HA & 327 & 19.25 & 146 & NA & 415 & 9.43 \\
\hline 25 & PA & 61 & 12.34 & 86 & HA & 327 & 10.41 & 147 & NA & 416 & 13.74 \\
\hline 26 & PA & 63 & 9.70 & 87 & HA & 328 & 16.24 & 148 & NA & 418 & 9.09 \\
\hline 27 & PA & 129 & 9.34 & 88 & HA & 377 & 13.91 & 149 & NA & 445 & 12.13 \\
\hline 28 & PA & 337 & 11.25 & 89 & HA & 397 & 16.18 & 150 & NA & 468 & 9.66 \\
\hline 29 & PA & 356 & 12.77 & 90 & HA & 407 & 9.49 & 151 & M1 & 15 & 9.79 \\
\hline 30 & PA & 367 & 14.56 & 91 & HA & 431 & 13.52 & 152 & M1 & 27 & 12.16 \\
\hline 31 & PA & 405 & 10.01 & 92 & HA & 492 & 9.49 & 153 & M1 & 37 & 14.66 \\
\hline 32 & PA & 554 & 14.67 & 93 & HA & 495 & 11.15 & 154 & M1 & 46 & 14.96 \\
\hline 33 & PA & 607 & 11.97 & 94 & HA & 496 & 10.62 & 155 & M1 & 101 & 13.28 \\
\hline 34 & PA & 684 & 12.20 & 95 & HA & 500 & 11.88 & 156 & M1 & 140 & 12.40 \\
\hline 35 & PA & 712 & 9.25 & 96 & HA & 503 & 12.76 & 157 & M1 & 142 & 11.31 \\
\hline
\end{tabular}


Table 1. Cont.

\begin{tabular}{|c|c|c|c|c|c|c|c|c|c|c|c|}
\hline Num & Pro $^{1}$ & $\operatorname{Pos}^{2}$ & Score & Num & Pro & Pos & Score & Num & Pro & Pos & Score \\
\hline 36 & HA & 40 & 9.42 & 97 & HA & 526 & 11.91 & 158 & M1 & 166 & 17.35 \\
\hline 37 & HA & 42 & 9.21 & 98 & HA & 530 & 11.26 & 159 & M1 & 205 & 11.09 \\
\hline 38 & HA & 45 & 11.92 & 99 & HA & 531 & 11.67 & 160 & M1 & 219 & 13.18 \\
\hline 39 & HA & 46 & 16.27 & 100 & HA & 534 & 12.77 & 161 & M1 & 224 & 23.52 \\
\hline 40 & HA & 53 & 9.87 & 101 & $\mathrm{NP}$ & 34 & 17.45 & 162 & M1 & 232 & 14.80 \\
\hline 41 & HA & 57 & 9.42 & 102 & $\mathrm{NP}$ & 77 & 12.39 & 163 & M1 & 242 & 19.59 \\
\hline 42 & HA & 65 & 10.99 & 103 & $\mathrm{NP}$ & 105 & 10.61 & 164 & M1 & 248 & 11.25 \\
\hline 43 & HA & 66 & 11.13 & 104 & NP & 373 & 14.73 & 165 & M2 & 13 & 13.66 \\
\hline 44 & HA & 79 & 12.71 & 105 & $\mathrm{NP}$ & 377 & 21.88 & 166 & M2 & 21 & 10.53 \\
\hline 45 & HA & 81 & 12.03 & 106 & NP & 482 & 19.71 & 167 & M2 & 97 & 15.79 \\
\hline 46 & HA & 84 & 10.27 & 107 & NA & 19 & 9.20 & 168 & NS1 & 77 & 10.59 \\
\hline 47 & HA & 91 & 17.33 & 108 & NA & 23 & 11.02 & 169 & NS1 & 80 & 12.48 \\
\hline 48 & HA & 96 & 14.98 & 109 & NA & 37 & 9.57 & 170 & NS1 & 81 & 12.55 \\
\hline 49 & HA & 102 & 9.04 & 110 & NA & 41 & 11.30 & 171 & NS1 & 82 & 12.01 \\
\hline 50 & HA & 112 & 12.67 & 111 & NA & 42 & 9.33 & 172 & NS1 & 83 & 14.52 \\
\hline 51 & HA & 114 & 19.46 & 112 & NA & 47 & 10.12 & 173 & NS1 & 84 & 10.21 \\
\hline 52 & HA & 115 & 9.66 & 113 & NA & 48 & 11.23 & 174 & NS1 & 172 & 14.21 \\
\hline 53 & HA & 121 & 10.42 & 114 & NA & 49 & 10.85 & 175 & NS1 & 179 & 11.18 \\
\hline 54 & HA & 124 & 10.28 & 115 & NA & 50 & 9.14 & 176 & NS1 & 197 & 9.32 \\
\hline 55 & HA & 131 & 12.31 & 116 & NA & 52 & 12.38 & 177 & NS1 & 212 & 14.19 \\
\hline 56 & HA & 142 & 12.01 & 117 & NA & 52 & 10.34 & 178 & NEP & 14 & 13.01 \\
\hline 57 & $\mathrm{HA}$ & 163 & 10.07 & 118 & NA & 52 & 9.75 & 179 & NEP & 22 & 15.38 \\
\hline 58 & $\mathrm{HA}$ & 164 & 9.03 & 119 & NA & 53 & 9.03 & 180 & NEP & 40 & 10.28 \\
\hline 59 & HA & 167 & 14.22 & 120 & NA & 58 & 11.05 & 181 & NEP & 60 & 9.17 \\
\hline 60 & HA & 173 & 12.81 & 121 & NA & 60 & 9.34 & 182 & NEP & 100 & 10.58 \\
\hline 61 & HA & 174 & 10.16 & 122 & NA & 63 & 9.44 & 183 & NEP & 115 & 11.10 \\
\hline
\end{tabular}

The AA factors and RF method screened 183 signature positions, some of which are reported to be associated with the mechanism of interspecies transmission. All of the residues were useful, not only for constructing the prediction model but also for further investigating the molecular mechanisms underlying the interspecies transmission of AIVs.

\subsection{Performance of the Prediction Model}

The performances of the four classifiers are presented as boxplots in Figure $1 \mathrm{~b}$. The results were obtained from 100 repeats of 10-fold cross-validation. The area under the curve (AUC) medians in the support vector machine (SVM) and RF classifiers were almost 1 . The AUC was clearly lower in the $k$-nearest neighbor (KNN) classifier, possibly because of the nonlinear prediction rules. Although the naïve Bayes (NB) classifier achieved a similar AUC score to the SVM classifier, its performance was poorer and less stable than those of the SVM and RF classifiers. Considering the complexity of the computation, the SVM classifier was selected as the optimal machine learning model for predicting avian-to-human transmission of AIVs.

\subsection{Contributions of the AA Factors}

The AIV strains were characterized by five AA factors. To understand the mechanism of interspecies transmission, the performance of the SVM classifier was calculated for all combination patterns of these AA factors. The result reveals the importance of the five AA factors. Most of the stable performances of the SVM classifier were contributed by AA Factor 3 or AA Factor 4 (Figure 2a). Notably, the median AUC values were almost 1 and remained stable under AA Factor 3 or AA Factor 4 alone. The SVM classifiers were unstable under AA Factor 1, AA Factor 2, and AA Factor 5 alone. Moreover, AA Factor 3 yielded a slightly better result than AA Factor 4 . These results indicate an 
important role for AA Factor 3 in the avian-to-human transmission of AIVs. Therefore, AA Factor 3 was employed in further analysis.
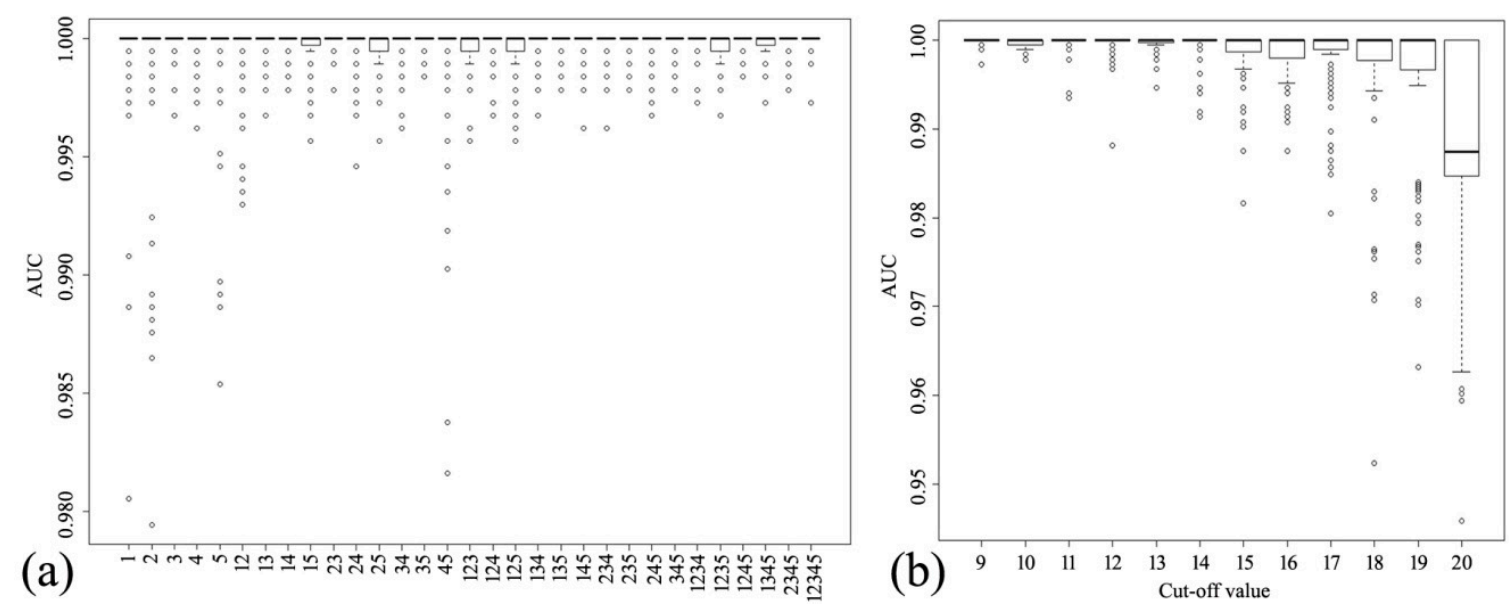

Figure 2. Contributions of AA factors and different mutation sets. (a) Performance of SVM classifier for different combinations of the five AA factors. The $x$ and $y$ coordinates denote the 31 combination patterns and the AUC values (from 0 to 1), respectively. Along the $x$ axis, '13' denotes that the set of 183 amino acid residues was transformed using AA Factor 1 and AA Factor 3 together, for example. (b) Contributions of mutation positions for different cutoff values (range 9-20). The y coordinate shows the AUC values.

\subsection{Contributions of Mutation Positions at Different Cutoff Values}

As mentioned above, 183 mutation sites survived a cutoff value of 9 . To further explore the mechanism of interspecies transmission, we should reduce the range of crucial positions. To this end, the cutoff value was incremented in steps of 1 (thereby decreasing the number of mutation sites), and the performance of the SVM classifier was calculated with the five AA factors. As shown in Figure $2 b$, the SVM classifier achieved stable and high performance at cutoffs up to 14 . The SVM classifier destabilized at higher cutoffs.

Considering the results under AA factor combinations and cutoff values, the performance of the SVM classifier with AA Factor 3 alone was assessed for different cutoffs. In this situation, the SVM classifier performed stably and well up to a cutoff of 13 (Figure 3a). The analysis results confirm that 13 is the extreme cutoff, giving 50 signature positions (Figure $3 b$ ). This set was regarded as the minimal mutation position set for predicting AIVs. We transformed these 50 signature residues using AA Factor 3 alone, and obtained the patterns of the human-origin AIVs (positive samples) by the multidimensional scaling method (Table S2). The resulting clusters are shown in Figure 3c. Cluster 1 comprises three H9N2 viruses (A/Hong Kong/1073/1999; A/Korea/KBNP-0028/2000; A/Bangladesh/0994/2011), two H7N3 viruses (A/Canada/rv504/2004; A/Mexico/InDRE7218/2012), two H7N7 viruses (A/Netherlands/219/2003; A/Italy/3/2013), and one H5N1 virus (A/Hong Kong/482/1997). Cluster 2 includes only H5N1 viruses isolated from 2003 to 2015. Cluster 3 is composed of H7N9 viruses, two H5N6 viruses (A/Yunnan/14563/2015; A/Yunnan/0127/2015), and two H9N2 viruses (A/Hong Kong/308/2014; A/Hunan/44558/2015). The distribution of the human-origin AIVs suggests that three molecular patterns of avian-to-human interspecies transmission emerge in nature. 


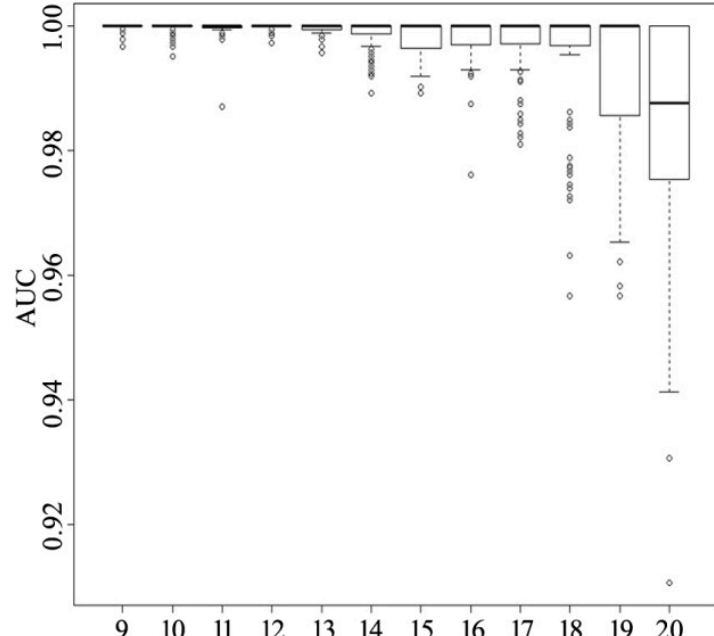

(a)

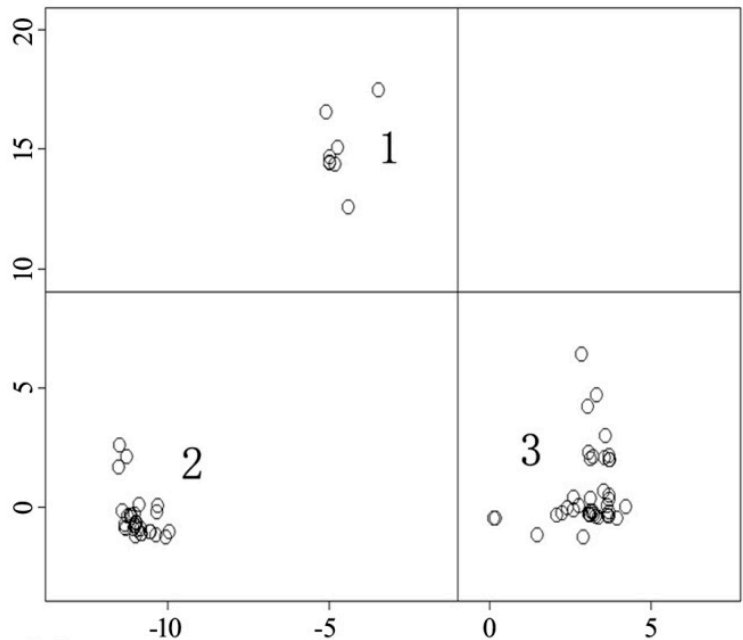

(c)
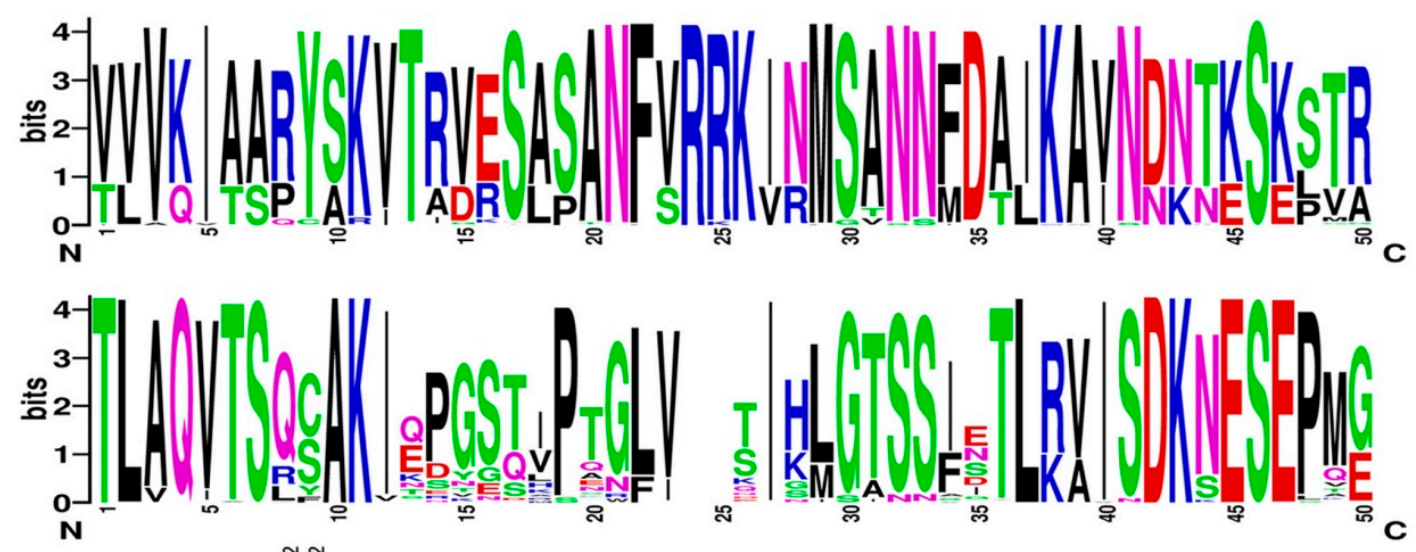

(b)

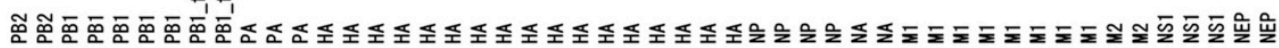

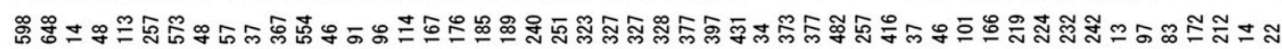

Figure 3. Minimal amino acid set for predicting AIVs. (a) Contributions of reduced mutation position sets. The $x$ and $y$ coordinates denote the cutoff (range 9-20) and the AUC values (range 0-1), respectively.

(b) Profiles of 50 signature positions from human-origin (top) and avian-origin (bottom) AIVs.

(c) Three patterns of human-origin AIVs clustered by the multidimensional scaling (MDS) method.

\section{Discussion}

Avian influenza viruses can cross the species barrier, potentially causing a human pandemic. In this paper, human AIV transmission was predicted by a machine learning model with excellent performance (namely, SVM). We firstly screened 183 mutation positions in 11 viral proteins after ranking them by random forest (RF). Most of the screened amino acid positions locate in the important functional regions of receptor binding, fusion peptides, intracellular transport, protein active sites, or virus assembly [26-37]. Some of the residues at these positions have been related to interspecies transmission in earlier reports, such as HA102-HA290 [26,27], H163 [28], HA91, HA96, HA328, HA377 and HA397 [29], HA327 [30], NA52 [31], and PB2 627 [33]. The signature positions guarantee the accuracy of the classifier and are biologically meaningful, which will benefit epidemic surveillance and further studies on interspecies AIV transmission. The proposed method provides important clues for future surveillance and is a useful pre-screening tool for phenotype screening in high-level biological safety laboratories. 
The signature positions related with the phenotype of interspecies transmission were screened by the method of random forest. Some yielded a modest score (PB2 627, for example). PB2 E627K was firstly identified in a mouse model [33] and found in the protein of other human-origin avian influenza viruses [12]. In part of the PB2 protein of the human seasonal influenza virus from the public database, PB2 E627 still existed. It is possible that the mutation PB2 E627K is not a strong marker for interspecies transmission, which is consistent with our results. In the future, we need to update the model with new molecular evidence in the field of virology and with more powerful technology in the field of machine learning.

Amino acid mutations in the HA protein are essential for AIV transmission in mammals [21], but mutations in other viral proteins are also necessary $[19,20]$. Mutations of different proteins introduce synergy and nonlinearity in interspecies transmission. This concept was supported by the present study. Specifically, the linear classifier (the KNN model) showed poor predictive performance on the initial set of 183 signature positions. Moreover, the minimal signature position set was 50 amino acid long and distributed among different viral proteins. This synergistic effect should be notable in further study.

The molecular characteristics of AA Factor 3 are related to molecular size or volume with high factor coefficients of bulkiness, residue volume, average volume of buried residues, side chain volume, and molecular weight [22]. Molecular size or volume is strong related with the binding of biology molecules, such as viral surface protein, host receptor, enzyme, and substrate. In this paper, the AA Factor 3 makes an important contribution to the prediction in terms of high accuracy, which agrees with previous results concerning the receptor binding of viral surface protein [26-28], enzyme activity of viral neuraminidase [32], and RNA binding of viral polymerase [34]. The slightly poor performance of other factors may suggest that host receptor binding, virus partial release triggered by viral neuraminidase, and viral polymerase activity play key roles for the interspecies transmission of avian influenza virus.

The patterns of human-origin AIVs were clarified by the MDS method. Cluster 1 was composed of one H5N1 virus from 1997; three H9N2 viruses from 1999, 2000, and 2011; two H7N3 viruses from 2004 and 2012; and two H7N7 viruses from 2003 and 2013. Cluster 2 contained only H5N1 viruses isolated from 2003 to 2015. Cluster 3 contained H7N9 viruses, two H5N6 viruses from 2015, and two H9N2 viruses from 2014 and 2015. The distribution of human-origin AIVs implies that three molecular patterns of avian-to-human interspecies transmission have emerged. Further investigations on the appearance of novel patterns should be undertaken in future.

The proposed method is applicable to other infectious pathogens that can cross species barriers. As deep learning technology develops, powerful methods that omit feature selection and complex computations might emerge. To better understand the interspecies transmission mechanism of AIVs, the prediction model could be supplemented with information on the host's genetic background [38].

\section{Materials and Methods}

\subsection{Dataset}

The avian- and human-origin AIVs were collected from the EpiFlu public database (http:/ / platform.gisaid.org/epi3/frontend) and processed using multiple public bioinformatics tools and algorithms (Figure 4). The details of each procedure are described below.

Step 1: In total, 6305 avian-origin and 644 human-origin AIV strains were obtained from the public influenza virus database. The strains were isolated between January 1996 and February 2016. GISAID deposits high-quality genomic sequences along with their clinical information.

Step 2: Our prediction classifiers were based on eleven viral proteins (PB2, PB1, PBI-F2, PA, HA, NP, NA, M1, M2, NS1, and NEP) with reported roles in interspecies transmission. AIV strains lacking any of these 11 protein sequences in the GISAID database, and strains without subtype information, were excluded in this step. 
Step 3: The amino acid residues in the 11 proteins were numbered by the multiple sequence alignment tool MUSCLE [39], using the seasonal human H3 subtype virus as the reference. This step eliminated strains lacking more than 3 amino acids at any protein terminal. The missing residues were replaced by the corresponding residues in the protein sequence with highest identity.

Step 4: To reduce redundancy in the dataset, the AIV strains should differ by at least one amino acid. The amino acid sequences were compared using the CD-Hit tool [40].

Step 5: If the genome sequences of the avian-origin and human-origin AIV strains share high identity, the interspecies transmission capabilities of the avian-origin strains are ambiguous. Therefore, this step eliminated avian-origin strains in which any nucleotide sequence of the eight genome segments shared $>97 \%$ identity with that of the human-origin strains. The elimination was performed by the BLAST + tool [41].

Step 6: Ambiguous amino acid residues such as ' $\mathrm{X}$ ' and ' $\mathrm{B}$ ' were replaced by the corresponding residues in the protein sequence with highest identity.

The final dataset for predicting AIV interspecies transmission contained 429 positive samples (human-origin AIVs) and 440 negative samples (avian-origin AIVs). All of these strains are listed in Table S1.
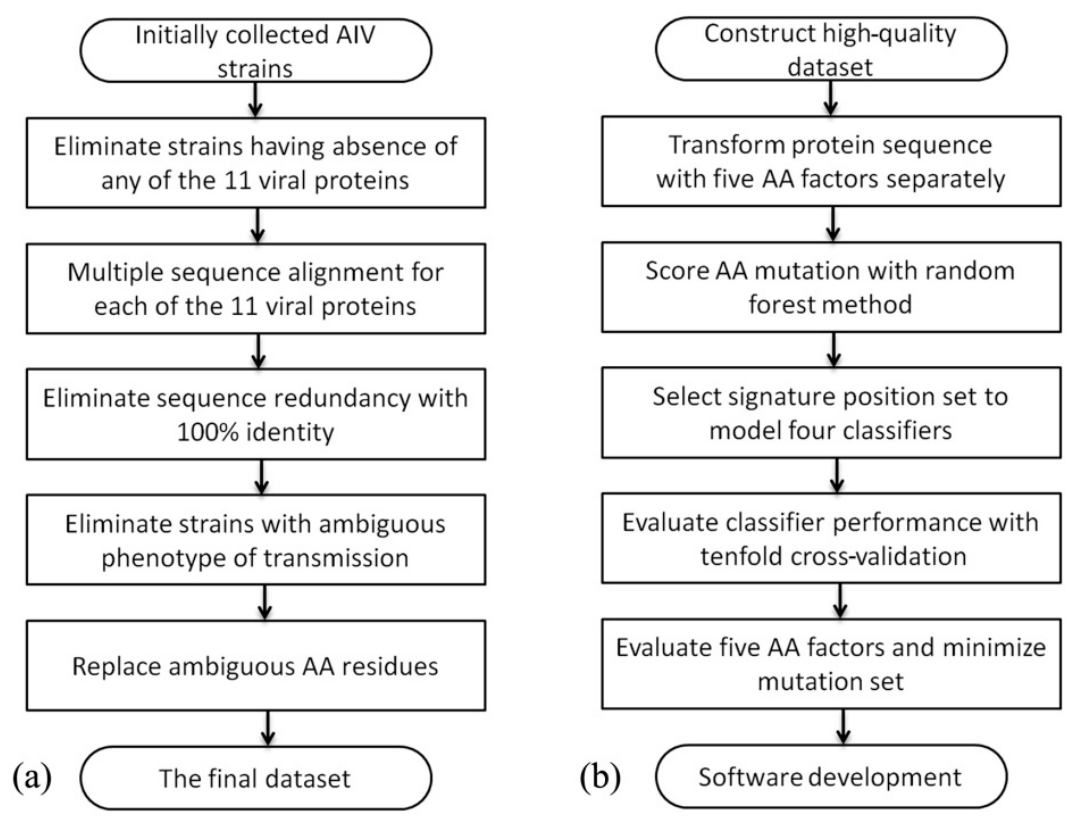

Figure 4. Flowchart of methods used in this paper. (a) High-quality dataset construction; (b) Machine learning algorism.

\subsection{Recognition of Signature Positions}

The random forest method is very popularly used for feature selection of prediction problems and can rank the importance of the features in a large scale to discriminate the different categories. The signature positions in the 11 viral proteins were recognized by the RF method (RF, https://cran.r-project.org/web/packages/randomForest/index.html). In each strain, the 11 proteins were concentrated in the following order: PB2 $>$ PB1 $>$ PB1-F2 $>$ PA $>$ HA $>$ $\mathrm{NP}>\mathrm{NA}>\mathrm{M} 1>\mathrm{M} 2>\mathrm{NS1}>\mathrm{NEP}$. The proteins with the length of 4620 amino acids were then transformed into numerical sequences of the amino acid factor. Any deletions or insertions in the protein were replaced by zeros. The strains were processed sequentially and accumulated into the total dataset, which was input to the RF. The positive samples (human-origin AIVs) and negative samples (avian-origin AIVs) were classified by their importance scores at each amino acid position. As the classification was based on five factors, the final importance score at each position was the sum 
of five calculations. Therefore, highly scoring positions were important for distinguishing positive and negative samples. These high scorers were regarded as important amino acid mutations in the interspecies transmission of AIVs. Breiman's random forest algorithm was used as default.

\subsection{Constructing the Classifier Model}

The machine learning method can solve the classification problem and the numeric features of the positive and negative samples are essential for classification. After screening the signature positions as mentioned above, each strain was represented by an amino acid residue set. These amino acid sets were again transformed into numerical sequences of the five AA factors. Each strain was represented as a numeric vector of length $5 \mathrm{~N}$, where $\mathrm{N}$ is the number of amino acids in an amino residue set. The interspecies AIV transmission was then predicted by four popular machine learning models that are widely used in bioinformatics and computational biology: (1) support vector machine (SVM, https://cran.r-project.org/web/packages/e1071/index.html), (2) random forest (RF, https://cran.r-project.org/web/packages/randomForest/index.html), (3) naïve Bayes (NB, https://cran.r-project.org/web/packages/e1071/index.html), and (4) k-nearest neighbor (KNN, https://cran.r-project.org/web/packages/class/index.html). The present prediction task is a two-class classification problem (in which human-origin and avian-origin AIVs are classified as positive and negative, respectively). The four classifiers were implemented in the $\mathrm{R}$ environment and related packages.

The SVM classifier performs the classification in a high-dimensional feature space, which was transferred from the input feature vector with the kernel function. If the samples from two categories were partly overlapped in the original feature space, the SVM will have good performance. In this paper, the optimal hyperplane is determined with the regularization parameter $\mathrm{C}(\mathrm{C}=1)$ and the radial basis function (RBF) as default. The RF classifier is an ensemble of many decision trees. Each tree is fully grown using part of the samples in the training dataset selected with the bootstrap technique. The NB is constructed based on the Bayes theorem. Both RF and NB were implemented with the default parameter in the package. The KNN classifier is a nonparametric method to determine a sample category by a majority vote of its neighbors; the number of neighbors in this paper was set to be $3(k=3)$.

\subsection{Evaluating the Performance of Different Classifiers}

The four classifiers were trained on 387 positive samples and 396 negative samples randomly selected from the AIV dataset. The remaining $10 \%$ of samples ( 42 positive and 44 negative samples) were reserved as an independent test dataset for assessing the performances of the classifiers. The classifier performances were evaluated by 10-fold cross-validation and the receiver operating characteristic (ROC) curve. The area under the ROC curve (AUC) reveals the optimal parameters in the four classifiers. To compare the classifier performances, we repeated the evaluation process 100 times and plotted the distributions of the resulting AUC values. The ROC curve relates the true and false positive rates, where both rates range from 0 to 1 . The AUC was calculated by the 'ROCR' package in $\mathrm{R}$ (https://cran.r-project.org/web/packages/ROCR/index.html). As both rates range from 0 to 1, AUC also ranges from 0 to 1 . A higher AUC value denotes a higher performance of the classifier. The human-origin AIVs were shown by the multidimensional scaling method in R (MDS, https:/ / cran.r-project.org/web/packages/MASS/index.html) and the amino acid profile was drawn by the WebLogo server (http:/ / weblogo.berkeley.edu/logo.cgi).

\subsection{Prediction Software}

By integrating the features at the signature positions with the best-performing classifier, we constructed a software program for predicting avian-to-human transmission of AIVs (delivery by request). 
Supplementary Materials: Table S1: AIV Strains in the final dataset, Table S2: Human-origin AIVs clustered by the MDS method.

Author Contributions: Conceptualization, X.Q. and Z.K.; Methodology, X.Q. and G.F.; Software, Y.W.; Writing-review and editing, Z.K.

Funding: This research was funded by the Chinese National Natural Science Foundation (61379059, $61472372,61632002)$.

Acknowledgments: We would like to acknowledge the originating and submitting laboratories of the viral sequences from GISAID's EpiFlu Database. We thank Leonie Pipe for manuscript editing.

Conflicts of Interest: The authors declare no conflict of interest.

\section{References}

1. Webster, R.G.; Bean, W.J.; Gorman, O.T.; Chambers, T.M.; Kawaoka, Y. Evolution and ecology of influenza A viruses. Microbiol. Rev. 1992, 56, 152-179. [CrossRef] [PubMed]

2. Xu, X.; Subbarao, K.; Cox, N.J.; Guo, Y. Genetic characterization of the pathogenic influenza A/Goose/Guangdong/1/96 (H5N1) virus: Similarity of its hemagglutinin gene to those of H5N1 viruses from the 1997 outbreaks in Hong Kong. Virology 1999, 261, 15-19. [CrossRef] [PubMed]

3. Claas, E.C.J.; Osterhaus, A.D.; van Beek, R.; De Jong, J.C.; Rimmelzwaan, G.F.; Senne, D.A.; Krauss, S.; Shortridge, K.F.; Webster, R.G. Human influenza A H5N1 virus related to a highly pathogenic avian influenza virus. Lancet 1998, 351, 472-477. [CrossRef]

4. Subbarao, K.; Klimov, A.; Katz, J.; Regnery, H.; Lim, W.; Hall, H.; Perdue, M.; Swayne, D.; Bender, C.; Huang, J.; et al. Characterization of an avian influenza A (H5N1) virus isolated from a child with a fatal respiratory illness. Science 1998, 279, 393-396. [CrossRef] [PubMed]

5. Chen, H.; Smith, G.J.; Li, K.S.; Wang, J.; Fan, X.H.; Rayner, J.M.; Vijaykrishna, D.; Zhang, J.X.; Zhang, L.J.; Guo, G.T.; et al. Establishment of multiple sublineages of H5N1 influenza virus in Asia: Implications for pandemic control. Proc. Natl. Acad. Sci. USA 2006, 103, 2845-2850. [CrossRef] [PubMed]

6. Li, K.S.; Guan, Y.; Wang, J.; Smith, G.J.; Xu, K.M.; Duan, L.; Rahardjo, A.P.; Puthavathana, P.; Buranathai, C.; Nguyen, T.D.; et al. Genesis of a highly pathogenic and potentially pandemic H5N1 influenza virus in eastern Asia. Nature 2004, 430, 209-213. [CrossRef] [PubMed]

7. Zhu, Q.Y.; Qin, E.D.; Wang, W.; Yu, J.; Liu, B.H.; Hu, Y.; Hu, J.F.; Cao, W.C. Fatal infection with influenza A (H5N1) virus in China. N. Engl. J. Med. 2006, 354, 2731-2732. [CrossRef] [PubMed]

8. Shu, Y.L.; Yu, H.J.; Li, D.X. Lethal avian influenza A (H5N1) infection in a pregnant woman in Anhui province, China. N. Engl. J. Med. 2006, 354, 1421-1422. [CrossRef] [PubMed]

9. Peiris, M.; Yuen, K.Y.; Leung, C.W.; Chan, K.H.; Ip, P.L.; Lai, R.W.; Orr, W.K.; Shortridge, K.F. Human infection with influenza H9N2. Lancet 1999, 354, 916-917. [CrossRef]

10. Butt, K.M.; Smith, G.J.; Chen, H.; Zhang, L.J.; Leung, Y.H.; Xu, K.M.; Lim, W.; Webster, R.G.; Yuen, K.Y.; Malik Peiris, J.S.; et al. Human infection with an avian H9N2 influenza A virus in Hong Kong in 2003. J. Clin. Microbiol. 2005, 43, 5760-5767. [CrossRef] [PubMed]

11. Fouchier, R.A.; Schneeberger, P.M.; Rozendaal, F.W.; Broekman, J.M.; Kemink, S.A.; Munster, V.; Kuiken, T.; Rimmelzwaan, G.F.; Schutten, M.; van Doornum, G.J.J.; et al. Avian influenza A virus (H7N7) associated with human conjunctivitis and a fatal case of acute respiratory distress syndrome. Proc. Natl. Acad. Sci. USA 2004, 101, 1356-1361. [CrossRef] [PubMed]

12. Gao, R.; Cao, B.; Hu, Y.; Feng, Z.; Wang, D.; Hu, W.; Chen, J.; Jie, Z.; Qiu, H.; Xu, K.; et al. Human infection with a novel avian-origin influenza A (H7N9) virus. N. Engl. J. Med. 2013, 368, 1888-1897. [CrossRef] [PubMed]

13. Cao, H.F.; Liang, Z.H.; Feng, Y.; Zhang, Z.N.; Xu, J.; He, H. A confirmed severe case of human infection with avian-origin influenza H7N9: A case report. Exp. Ther. Med. 2015, 9, 693-696. [CrossRef] [PubMed]

14. Zeng, X.; Liu, L.; Lv, L.; Zou, Q. Prediction of potential disease-associated microRNAs using structural perturbation method. Bioinformatics 2018. [CrossRef] [PubMed]

15. Zeng, X.; Zhang, X.; Zou, Q. Integrative approaches for predicting microRNA function and prioritizing disease-related microRNA using biological interaction networks. Brief. Bioinform. 2016, 17, 193-203. [CrossRef] [PubMed] 
16. Gang, F.; Zhang, S.; Dong, Y. Optimizing DNA assembly based on statistical language modelling. Nucleic Acids Res. 2017, 45, e182. [CrossRef]

17. Zeng, X.; Liao, Y.; Liu, Y.; Zou, Q. Prediction and validation of disease genes using HeteSim Scores. IEEE/ACM Trans. Comput. Biol. Bioinform. 2017, 14, 687-695. [CrossRef] [PubMed]

18. Zeng, X.; Lin, W.; Guo, M.; Zou, Q. A comprehensive overview and evaluation of circular RNA detection tools. PLoS Comput. Biol. 2017, 13, e1005420. [CrossRef] [PubMed]

19. Herfst, S.; Schrauwen, E.J.; Linster, M.; Chutinimitkul, S.; de Wit, E.; Munster, V.J.; Sorrell, E.M.; Bestebroer, T.M.; Burke, D.F.; Smith, D.J.; et al. Airborne transmission of influenza A/H5N1 virus between ferrets. Science 2012, 336, 1534-1541. [CrossRef] [PubMed]

20. Imai, M.; Watanabe, T.; Hatta, M.; Das, S.C.; Ozawa, M.; Shinya, K.; Zhong, G.; Hanson, A.; Katsura, J.; Watanabe, S.; et al. Experimental adaptation of an influenza H5 HA confers respiratory droplet transmission to a reassortant H5 HA/H1N1 virus in ferrets. Nature 2012, 486, 420-428. [CrossRef] [PubMed]

21. Glaser, L.; Stevens, J.; Zamarin, D.; Wilson, I.A.; García-Sastre, A.; Tumpey, T.M.; Basler, C.F.; Taubenberger, J.K.; Palese, P. A single amino acid substitution in 1918 influenza virus hemagglutinin changes receptor binding specificity. J. Virol. 2005, 79, 11533-11536. [CrossRef] [PubMed]

22. Atchley, W.R.; Zhao, J.; Fernandes, A.D.; Drüke, T. Solving the protein sequence metric problem. Proc. Natl. Acad. Sci. USA 2005, 102, 6395-6400. [CrossRef] [PubMed]

23. Kou, Z.; Lei, F.; Wang, S.; Zhou, Y.; Li, T. Molecular patterns of avian influenza A viruses. Chin. Sci. Bull. 2008, 53, 2002-2007. [CrossRef]

24. Qiang, X.; Kou, Z. Prediction of interspecies transmission for avian influenza A virus based on a back-propagation neural network. Math. Comput. Model. 2010, 52, 2060-2065. [CrossRef]

25. Wang, J.; Kou, Z.; Duan, M.; Ma, C.; Zhou, Y. Using amino acid factor scores to predict avian-to-human transmission of avian influenza viruses: A machine learning study. Protein Pept. Lett. 2013, 20, 1115-1121. [CrossRef] [PubMed]

26. Stevens, J.; Corper, A.L.; Basler, C.F.; Taubenberger, J.K.; Palese, P.; Wilson, I.A. Structure of the uncleaved human H1 hemagglutinin from the extinct 1918 influenza virus. Science 2004, 303, 1866-1870. [CrossRef] [PubMed]

27. Hulse, D.J.; Webster, R.G.; Russell, R.J.; Perez, D.R. Molecular determinants within the surface proteins involved in the pathogenicity of H5N1 influenza viruses in chickens. J. Virol. 2004, 78, 9954-9964. [CrossRef] [PubMed]

28. Mishin, V.P.; Novikov, D.; Hayden, F.G.; Gubareva, L.V. Effect of hemagglutinin glycosylation on influenza virus susceptibility to neuraminidase inhibitors. J. Virol. 2005, 79, 12416-12424. [CrossRef] [PubMed]

29. Chen, J.; Skehel, J.J.; Wiley, D.C. N- and C-terminal residues combine in the fusion-pH influenza hemagglutinin HA (2) subunit to form an N cap that terminates the triple-stranded coiled coil. Proc. Natl. Acad. Sci. USA 1999, 96, 8967-8972. [CrossRef] [PubMed]

30. Schrauwen, E.J.A.; de Graaf, M.; Herfst, S.; Rimmelzwaan, G.F.; Osterhaus, A.D.M.E.; Fouchier, R.A.M. Determinants of virulence of influenza A virus. Eur. J. Clin. Microbiol. Infect. Dis. 2014, 33, 479-490. [CrossRef] [PubMed]

31. Zhou, H.; Yu, Z.; Hu, Y.; Tu, J.; Zou, W.; Peng, Y.; Zhu, J.; Li, Y.; Zhang, A.; Yu, Z.; et al. The special neuraminidase stalk-motif responsible for increased virulence and pathogenesis of H5N1 influenza A virus. PLoS ONE 2009, 4, e6277. [CrossRef] [PubMed]

32. Barman, S.; Adhikary, L.; Chakrabarti, A.K.; Bernas, C.; Kawaoka, Y.; Nayak, D.P. Role of transmembrane domain and cytoplasmic tail amino acid sequences of influenza a virus neuraminidase in raft association and virus budding. J. Virol. 2004, 78, 5258-5269. [CrossRef] [PubMed]

33. Hatta, M.; Gao, P.; Halfmann, P.; Kawaoka, Y. Molecular basis for high virulence of Hong Kong H5N1 influenza A viruses. Science 2001, 293, 1840-1842. [CrossRef] [PubMed]

34. Perez, D.R.; Donis, R.O. Functional analysis of PA binding by influenza a virus PB1: Effects on polymerase activity and viral infectivity. J. Virol. 2001, 75, 8127-8136. [CrossRef] [PubMed]

35. Iwatsuki-Horimoto, K.; Horimoto, T.; Noda, T.; Kiso, M.; Maeda, J.; Watanabe, S.; Muramoto, Y.; Fujii, K.; Kawaoka, Y. The cytoplasmic tail of the influenza A virus M2 protein plays a role in viral assembly. J. Virol. 2006, 80, 5233-5240. [CrossRef] [PubMed] 
36. Bullido, R.; Gomez-Puertas, P.; Albo, C.; Portela, A. Several protein regions contribute to determine the nuclear and cytoplasmic localization of the influenza A virus nucleoprotein. J. Gen. Virol. 2000, 81, 135-142. [CrossRef] [PubMed]

37. Iwatsuki-Horimoto, K.; Horimoto, T.; Fujii, Y.; Kawaoka, Y. Generation of influenza A virus NS2 (NEP) mutants with an altered nuclear export signal sequence. J. Virol. 2004, 78, 10149-10155. [CrossRef] [PubMed]

38. Srivastava, B.; Błazejewska, P.; Heßmann, M.; Bruder, D.; Geffers, R.; Susanne, M.; Gruber, A.D.; Schughart, K. Host genetic background strongly influences the response to influenza A virus infections. PLoS ONE 2009, 4, e4857. [CrossRef] [PubMed]

39. Edgar, R.C. MUSCLE: multiple sequence alignment with high accuracy and high throughput. Nucleic Acids Res. 2004, 32, 1792-1797. [CrossRef] [PubMed]

40. Li, W.; Godzik, A. Cd-hit: A fast program for clustering and comparing large sets of protein or nucleotide sequences. Bioinformatics 2006, 22, 1658-1659. [CrossRef] [PubMed]

41. Altschul, S.; Gish, W.; Miller, W.; Myers, E.; Lipman, D. Basic local alignment search tool. J. Mol. Biol. 1990, 215, 403-410. [CrossRef]

Sample Availability: Samples of the compounds are not available from the authors. 